PROCEEDINGS OF THE

AMERICAN MATHEMATICAL SOCIETY

Volume 136, Number 9, September 2008, Pages 3351-3353

S 0002-9939(08)09481-1

Article electronically published on April 25, 2008

\title{
CONCORDANCE CROSSCAP NUMBERS OF KNOTS AND THE ALEXANDER POLYNOMIAL
}

\author{
CHARLES LIVINGSTON
}

(Communicated by Daniel Ruberman)

\begin{abstract}
For a knot $K$ the concordance crosscap number, $c(K)$, is the minimum crosscap number among all knots concordant to $K$. Building on work of G. Zhang, which studied the determinants of knots with $c(K)<2$, we apply the Alexander polynomial to construct new algebraic obstructions to $c(K)<2$. With the exception of low crossing number knots previously known to have $c(K)<2$, the obstruction applies to all but four prime knots of 11 or fewer crossings.
\end{abstract}

Every knot $K \subset S^{3}$ bounds an embedded surface $F \subset S^{3}$ with $F \cong \#_{n} P^{2}-B^{2}$ for some $n \geq 0$, where $P^{2}$ denotes the real projective plane. The crosscap number of $K, \gamma(K)$, is defined to be the minimum such $n$. The careful study of this invariant began with the work of Clark in [Cl]; other references include [HT, MY1. The study of the 4-dimensional crosscap number, $\gamma_{4}(K)$, defined similarly but in terms of $F \subset B^{4}$, appears in such articles as [MY2, Vi, Ya].

Gengyu Zhang $\mathrm{Zh}$ recently introduced a new knot invariant, the concordance crosscap number, $\gamma_{c}(K)$. This is defined to be the minimum crosscap number of any knot concordant to $K$. This invariant is the nonorientable version of the concordance genus, originally studied by Nakanishi $\mathrm{Na}$ and Casson $\mathrm{Ca}$, and later investigated in $\mathrm{Li}$.

In [Zh], Zhang presented an obstruction to $\gamma_{c}(K) \leq 1$ based on the homology of the 2 -fold branched cover of the knot, or equivalently, $\operatorname{det}(K)$. Inspired by her work, in this note we will observe that the obstruction found in Zh extends to one based on the Alexander polynomial of $K, \Delta_{K}(t)$, and the signature of $K, \sigma(K)$.

Theorem 1. Suppose $\gamma_{c}(K)=1$ and set $q=|\sigma(K)|+1$. For all odd prime power divisors $p$ of $q$, the $2 p$-cyclotomic polynomial $\phi_{2 p}(t)$ has odd exponent in $\Delta_{K}(t)$. Furthermore, every other symmetric irreducible polynomial $\delta(t)$ with odd exponent in $\Delta_{K}(t)$ satisfies $\delta(-1)= \pm 1$.

Proof. Any knot $K^{\prime}$ with $\gamma\left(K^{\prime}\right)=1$ bounds a Mobius band and is thus a $(2, r)-$ cable of some knot $J$ for some odd $r$. If $K$ is concordant to $K^{\prime}$, then $\sigma(K)=$ $\sigma\left(K^{\prime}\right)= \pm(|r|-1)$; the signature $\sigma\left(K^{\prime}\right)$ is given by a formula of Shinohara [Sh] for the signature of 2 -stranded cable knots. It follows that $|\sigma(K)|=|r|-1$, so $|r|=|\sigma(K)|+1=q$.

According to a result of Seifert [Se, the Alexander polynomial of $K^{\prime}$ is given by $\Delta_{2, q}(t) \Delta_{J}\left(t^{2}\right)$, where $\Delta_{2, q}(t)$ is the Alexander polynomial of the $(2, q)$-torus knot.

Received by the editors May 11, 2007.

2000 Mathematics Subject Classification. Primary 57M25.

(C)2008 American Mathematical Society Reverts to public domain 28 years from publication 
A standard result states that $\Delta_{2, q}(t)=\frac{\left(t^{2 q}-1\right)(t-1)}{\left(t^{q}-1\right)\left(t^{2}-1\right)}=\frac{t^{q}+1}{t+1}$. This can be written as the product of cyclotomic polynomials,

$$
\Delta_{2, q}(t)=\prod_{p \mid q, p>1} \Phi_{2 p}(t) .
$$

Since $K$ is concordant to $K^{\prime}, K \#-K^{\prime}$ is slice, and thus has Alexander polynomial of the form $g(t) g\left(t^{-1}\right)$. That is, with $q=|\sigma(K)|+1$,

$$
\Delta_{K}(t) \Delta_{J}\left(t^{2}\right) \Delta_{2, q}(t)=g(t) g\left(t^{-1}\right) \text {. }
$$

We now make two observations: (1) Any symmetric irreducible polynomial has even exponent in $g(t) g\left(t^{-1}\right)$, and thus even exponent in $\Delta_{K}(t) \Delta_{J}\left(t^{2}\right) \Delta_{(2, q)}(t) ;(2)$ since $\Delta_{J}(t)$ is an Alexander polynomial, $\Delta_{J}(1)= \pm 1$, and thus $\left.\Delta_{J}\left(t^{2}\right)\right|_{t=-1}= \pm 1$.

By Lemma 2. $\Phi_{2 p}(-1)=p$ if $p$ is an odd prime power, and $\Phi_{2 p}(-1)= \pm 1$ if $p$ is an odd composite. Thus, for $p$ an odd prime power divisor of $q, \Phi_{2 p}(t)$ has odd exponent in $\Delta_{2, q}(t)$ and does not divide $\Delta_{J}\left(t^{2}\right)$, so has odd exponent in $\Delta_{K}(t)$. Any other irreducible factor of $\Delta_{K}(t)$ with odd exponent is either a factor $\delta(t)$ of $\Delta_{2, q}(t)$, and thus of the form $\Phi_{2 p}(t)$ with $p$ an odd composite (and so $\delta(-1)= \pm 1$ ), or else is not a factor of $\Delta_{2, q}(t)$ and so has odd exponent in $\Delta_{J}\left(t^{2}\right)$, and again must satisfy $\delta(-1)= \pm 1$. This completes the argument.

Lemma 2. The cyclotomic polynomial $\Phi_{2 p}(t)$ satisfies $\Phi_{2 p}(-1)=p$ if $p$ is an odd prime power and $\Phi_{2 p}(-1)= \pm 1$ if $p$ is an odd composite.

Proof. For an odd $r, h_{r}(t)=\frac{t^{r}+1}{t+1}$ satisfies $h_{r}(-1)=r$ by l'Hôpital's rule. We have that $h_{r}(t)$ is the product

$$
h_{r}(t)=\prod_{p \mid r, p>1} \Phi_{2 p}(t) .
$$

For $p$ a prime power, $s^{n}, \Phi_{2 p}(t)=\frac{t^{s^{n}+1}}{t^{s^{n-1}+1}}$, and so, again by l'Hôpital's rule, $\Phi_{2 s^{n}}(-1)=s$. Thus, the product

$$
\prod_{p \mid r, p>1, p \text { a prime power }} \Phi_{2 p}(-1)=r .
$$

It follows that all the other terms in the product expansion of $h_{r}(t)$ must equal \pm 1 when evaluated at $t=-1$, as desired.

Example. Theorem 1 is quite effective in ruling out $\gamma_{c}(K)=1$. For instance, there are 801 prime knots with 11 or fewer crossings. Of these, 51 are known to be topologically slice, and 23 are known to be concordant to a $(2, q)$-torus knot for some $q$ and thus have $\gamma_{c}=1$. Of the remaining 727 knots, all but four can be shown to have $\gamma_{c} \geq 2$. These four are $11 n_{45}$ and $11 n_{145}$, both of which are possibly slice, and $9_{40}$ and $11 n_{66}$, both of which are possibly concordant to the trefoil. Of the collection of 727 knots, Yasuhara's result [Ya] applies to show that 207 of them have 4 -ball crosscap number $\gamma_{4}(K) \geq 2$. The 4 -ball crosscap numbers of the rest are unknown.

As a second set of examples, consider knots $K$ with $\Delta_{K}(t)$ of degree 2 . It follows immediately from Theorem 1 that there are only two possibilities: either $\sigma(K)=0$ and $\Delta_{K}$ is reducible (an irreducible symmetric quadratic $f(t)$ cannot satisfy $f(1)= \pm 1$ and $f(-1)= \pm 1)$ or $\sigma(K)= \pm 2$ and $\Delta_{K}(t)=t^{2}-t+1$. 
We conclude with the further special case consisting of $(p, q, r)$-pretzel knots, $P(p, q, r)$, with $p, q$, and $r$ odd; some of these were studied in [Zh]. If we let $D=D(p, q, r)=p q+q r+r p$, then

$$
\Delta_{P(p, q, r)}(t)=\frac{D+1}{4} t^{2}-\frac{D-1}{2} t+\frac{D+1}{4},
$$

which has discriminant $-D$. Thus, by the previous argument we have:

Corollary 3. If $\gamma_{c}(P(p, q, r))=1$, then either $\sigma(P(p, q, r))=0$ and $D(p, q, r)=$ $-l^{2}$ for some integer $l$ or $\sigma(P(p, q, r))= \pm 2$ and $D(p, q, r)=3$.

These pretzel knots include some shown by Zhang [Zh] to have 4-dimensional crosscap number $\gamma_{4}(K)=1$.

\section{REFERENCES}

[Ca] A. Casson, personal correspondence, 1980.

[Cl] B. Clark, Crosscaps and knots, Internat. J. Math. Math. Sci. 1 (1978), no. 1, 113-123. MR0478131 (57:17620)

[HT] M. Hirasawa and M. Teragaito, Crosscap numbers of 2-bridge knots, Topology 45 (2006), no. 3, 513-530. MR2218754 (2007h:57005)

[Li] C. Livingston, The concordance genus of knots, Alg. and Geom. Top. 4 (2004), 1-22. MR2031909 (2005e:57023)

[MY1] H. Murakami and A. Yasuhara, Crosscap number of a knot, Pacific J. Math. 171 (1995), no. 1, 261-273. MR1362987 (96k:57006)

[MY2] H. Murakami and A. Yasuhara, Four-genus and four-dimensional clasp number of a knot, Proc. Amer. Math. Soc. 128 (2000), no. 12, 3693-3699. MR1690998 (2001b:57020)

[Na] Y. Nakanishi, A note on unknotting number, Math. Sem. Notes Kobe Univ. 9 (1981), 99-108. MR634000 (83d:57005)

[Se] H. Seifert, On the homology invariants of knots, Quart. J. Math., Oxford Ser. (2) 1, (1950), 23-32. MR0035436 (11:735b)

[Sh] Y. Shinohara, On the signature of knots and links, Trans. Amer. Math. Soc. 156 (1971), 273-285. MR0275415(43:1172)

[Vi] O. Viro, Positioning in codimension 2, and the boundary, Uspehi Mat. Nauk 30 (1975), 231-232. MR0420641 (54:8654)

[Ya] A. Yasuhara, Connecting lemmas and representing homology classes of simply connected 4-manifolds, Tokyo J. Math. 19 (1996), no. 1, 245-261. MR.1391941 (97g:57027)

[Zh] G. Zhang, Concordance crosscap number of a knot, Bulletin of the London Mathematical Society 39 (2007), 755-761. MR2365224

Department of Mathematics, Indiana University, 123 Rawles Hall, Bloomington, INDIANA 47405 